\title{
Inconsistent trends regarding the association between ABO blood groups and susceptibility to SARS-CoV-2 infections
}

Jasmin Junseo Lee ${ }^{1,2, *}$ (D) and Carol Guo ${ }^{1}$ (D)
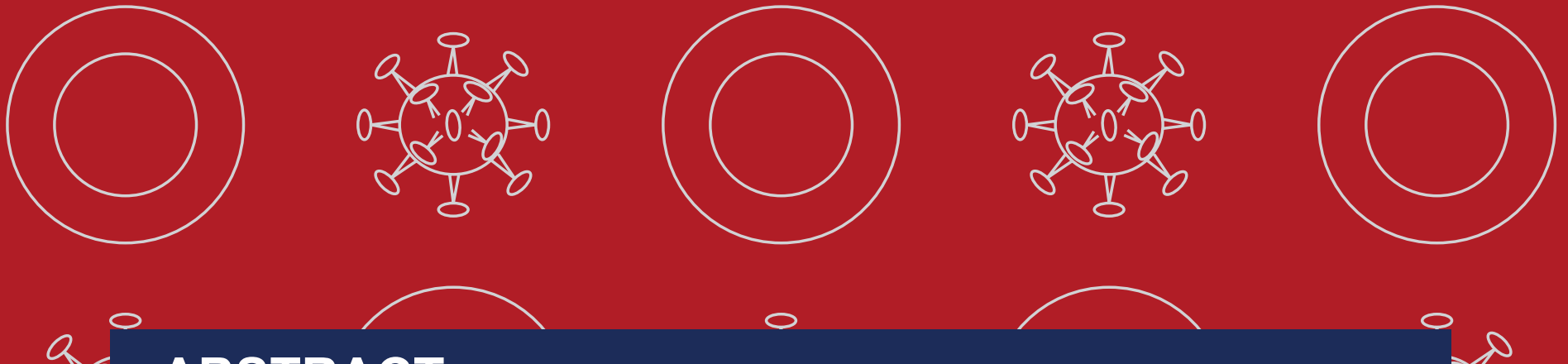

0
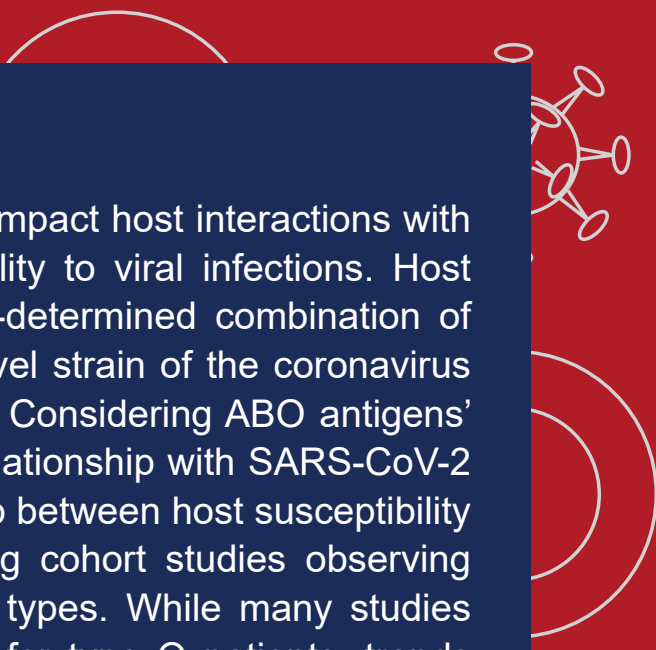

$\mathrm{ABO}$ antigens, produced from the $\mathrm{ABO}$ gene, are known to impact host interactions with various viruses. One characteristic is the host's susceptibility to viral infections. Host interaction with viral particles is altered by the blood type-determined combination of ABO antigens on the cellular surface. SARS-CoV-2 is a novel strain of the coronavirus family known to have structural similarities with SARS-CoV. Considering ABO antigens' association with SARS-CoV, studies have examined their relationship with SARS-CoV-2 as well. We reviewed current perspectives on the relationship between host susceptibility to SARS-CoV-2 infections and ABO antigens by examining cohort studies observing proportional differences between patients of varying blood types. While many studies indicated a higher risk of type $A$ patients and a lower risk for type $O$ patients, trends tended to vary per population. Consequently, we investigated the differences in study design between cohort studies that produced the variation in trends. Additionally, we examined genome-wide association studies that indicate a genetic association between the variables. We present the need for a standardized calculation method determining the significance of each blood group in its relationship with SARS-CoV-2 susceptibility per cohort.

Keywords: SARS-CoV-2, ABO blood group, COVID-19, disease susceptibility

Affiliations

${ }^{1}$ Human Biology Program, University of Toronto, Canada ${ }^{2}$ Korean Genomics Center (KOGIC), Ulsan National Institute of Science and Technology (UNIST), Republic of Korea
*Please direct correspondence to: Jasmin Junseo Lee

Email: jasmin.lee@mail.utoronto.ca

Published online on July 16, 2021 


\section{INTRODUCTION}

Severe acute respiratory syndrome coronavirus 2 (SARS-CoV-2) is a positive-strand RNA virus behind the COVID-19 pandemic that emerged in late December of $2019^{1}$. As of June 20th of 2021, the strain has infected more than $178,334,000$ people and taken the lives of more than $3,862,000$, resulting in a case fatality rate (CFR) of $\sim 2.17 \%{ }^{2}$. The CFR is comparably lower than that of other novel coronavirus outbreaks, such as MERS-CoV with a CFR of $34.4 \%$, and SARS-CoV with a CFR of $9.6 \%^{3,4}$. Alternatively, SARS-CoV-2 is more contagious than the other novel coronaviruses with a mean reproductive value (R0) of $\sim 3.28$, contributing to its widespread community transmission ${ }^{5,6}$.

Human blood types are classified by the phenotype of antigens present on the surface of red blood cells (RBC). There are two major blood type divisions: $A B O(A, B$, $A B$, and $O$ ) and Rh (Rh D-positive or Rh D-negative), determined by the $\mathrm{ABO}$ and Rh genes, respectively ${ }^{7}$. Based on polymorphic differences between antigen types, different blood types may experience varying levels of susceptibility to disease ${ }^{8}$. ABO antigens are present on epithelial cells as well, mediating contacts with pathogens. These antigens can be expressed as, or mimic the mechanisms of, receptors for pathogens to influence infection rates $^{9-11}$. Alternatively, Rh antigens are known to be highly immunogenic and able to stimulate immune responses ${ }^{12}$. For the scope of this paper, we will be limiting our discussion to $A B O$ groups.
The role of $A B O$ groups in the outcome of viral diseases has been assessed frequently in the past. However, the blood group associated with a significant disease outcome varies per virus. A cohort study of the West Nile virus of the flavivirus family placed type $A$ individuals at a higher risk for infection ${ }^{13}$. On the other hand, patients of $\mathrm{H} 3 \mathrm{~N} 2$ of the orthomyxoviridae family were more likely to be type B compared to other types ${ }^{14}$. Concerning viruses of the coronavirus family, an analysis of a SARS-CoV outbreak in Hong Kong indicated that type $\mathrm{O}$ individuals had a significantly lower infection rate in contrast to non-O types ${ }^{15}$.

Furthermore, a study on SARS-CoV highlighted the ability of $A B O$ antigens to interfere with the binding interaction between the viral spike (S) protein and the host angiotensin-converting enzyme 2 (ACE2), blocking subsequent infection. Thus, polymorphism of $\mathrm{ABO}$ antigens may play a role in altering the transmission rates of the SARS-CoV ${ }^{16}$. Considering the structural similarities between SARS-CoV and SARS-CoV-2, it is no surprise that SARS-CoV-2 also uses the ACE2 receptor for viral entry (Figure 1) ${ }^{17,18}$. The resemblance between the two viruses suggests a possible influence from the $A B O$ gene in the interaction between SARSCoV-2 with host ACE2 ${ }^{19}$.

Nonetheless, the incomplete understanding of the novel coronavirus, as well as its rapidly changing genome has resulted in disputes regarding the nature of the interaction. In this review, we examine current

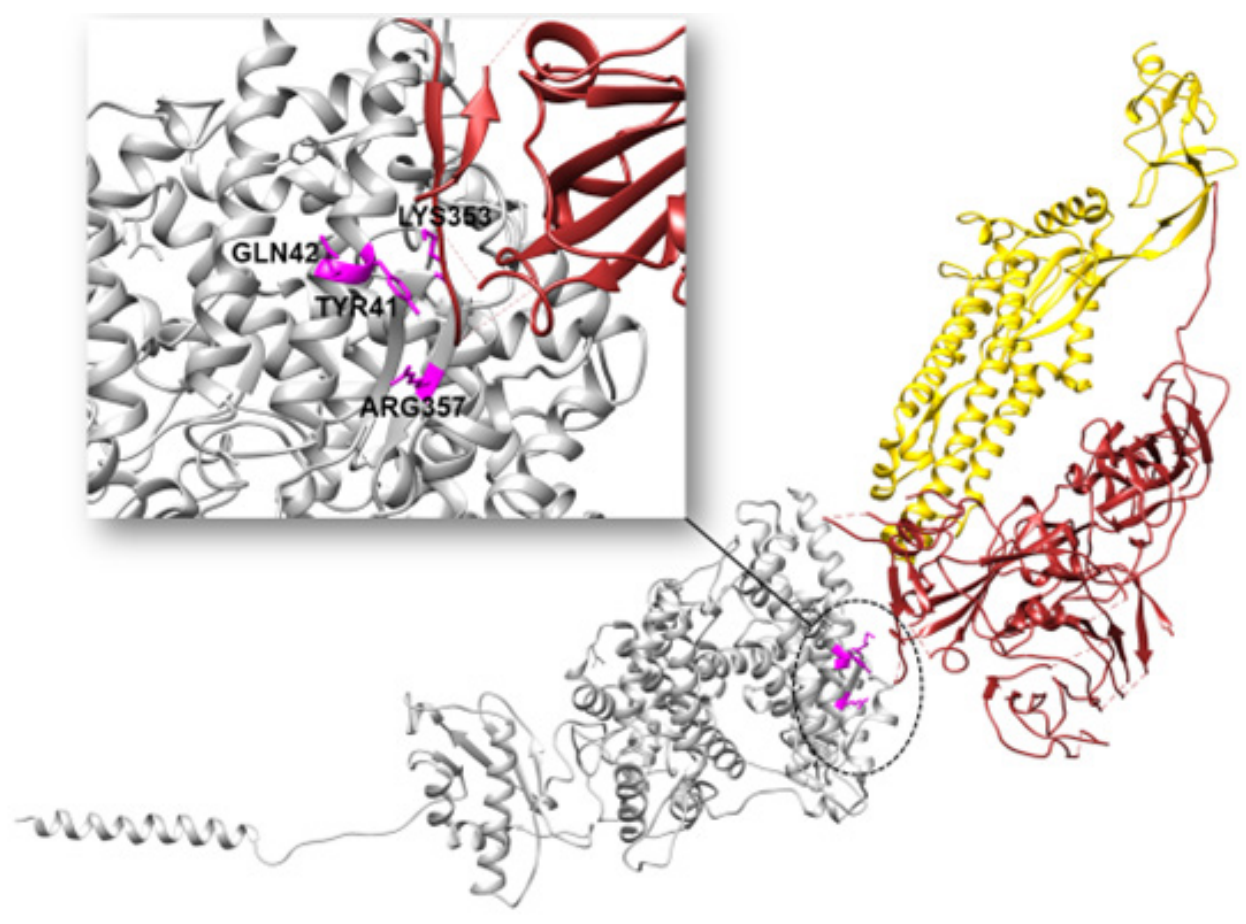

Figure 1 Binding between host ACE2 receptor and SARS-CoV-2 S protein. S1 subunit of SARS-CoV-2 S protein is in red, while the S2 subunit is in yellow. Host ACE2 receptor is in white. Binding regions of ACE2 are shown in magenta and are labelled. 


\begin{tabular}{|l|l|l|l|l|l|}
\hline \multirow{2}{*}{ Reference } & \multirow{2}{*}{ Cohort } & \multicolumn{3}{l|}{ P-value } \\
\cline { 3 - 6 } & & A & B & $\mathbf{0}$ & AB \\
\hline 21 & Wuhan Jinyintan Hospital, China & $<0.001$ & 0.240 & 0.291 & $<0.001$ \\
\cline { 2 - 6 } & Shenzhen Third People's Hospital, China & 1.000 & 0.142 & $<0.001$ & 0.001 \\
\hline 22 & Central Hospital of Wuhan, China & $\mathbf{0 . 0 1 7}$ & 0.891 & 0.696 & $<0.01$ \\
\hline 23 & Denmark & $<0.001$ & 0.091 & $<0.001$ & 0.011 \\
\hline 24 & First Hospital of Changsha, China & $\mathbf{0 . 0 0 6}$ & 0.707 & $\mathbf{0 . 0 1 8}$ & 0.270 \\
\hline 25 & $\begin{array}{l}\text { Hacettepe University School of Medicine } \\
\text { Hospital, Turkey }\end{array}$ & $<0.001$ & 0.155 & $\mathbf{0 . 0 0 1}$ & 0.364 \\
\hline 26 & Charles de Gaulle, French Navy Ship & $\mathbf{0 . 2 2}$ & 0.52 & $\mathbf{0 . 3 2}$ & 0.88 \\
\hline 29 & Five major hospitals in Massachusetts, US & 0.96 & $\mathbf{0 . 0 0 4}$ & 0.007 & 0.035 \\
\hline 34 & 33 regions of India & 0.452 & $\mathbf{0 . 0 3 7}$ & 0.206 & 0.179 \\
\hline 35 & Imam Khomeini Hospital Complex, Iran & 0.19 & 0.61 & $\mathbf{0 . 0 0 2}$ & 0.01 \\
\hline
\end{tabular}

Table 1 Comparison table of p-values between cohort studies for host susceptibility for SARS-CoV-2 vs. blood types.

perspectives on the correlation between $A B O$ blood groups and susceptibility to SARS-CoV-2 by reviewing publications from the onset of the pandemic. We identified the disagreement in trends between studies deriving from variations in their designs, therefore highlighting the need for a standardized method to calculate a blood group's significance.

\section{DISCUSSION}

\section{Evaluation of studies on the impact of $A B O$ antigens}

In March 2020, Zhao et al. published a paper proposing the impact of $A B O$ antigens on host susceptibility to SARS-CoV-2 infections. By comparing the distribution of patients' blood types across three hospitals in Wuhan and Shenzhen, China, the study deduced type Apatients to be more vulnerable $(P<0.001)$, while type $O$ patients are more protected $(\mathrm{P}<0.001)$, against COVID-1920. While the paper was initially criticized for its limitations (i.e., one-dimensional calculation via proportional data of patients), a volume of studies provided results from alternate populations that are consistent with the report by Zhao et al. ${ }^{21-25}$. While successful in identifying proportional differences between blood types of their patient cohorts, the studies could not understand the pathways causing the association.

A subsequent genome-wide association study (GWAS) was successful in identifying associations between the variables at a genomic level. The cohort study, focusing on populations from Italy and Spain, identified an association signal at 9q34.2 $(P<5 \times 10$ ${ }^{8}$ ), the ABO blood group locus. Additionally, a higher risk for type $A$ individuals and a lower risk for type O patients was found, consistent with previous studies26. Furthermore, the commercial DNA testing company AncestryDNA conducted a GWAS via genetic data from the AncestryDNA database, resulting in a robust sample size of 500,000 . The study identified a significant association at the ABO locus $(P<0.05)$, along with three novel loci in association with viral replications $\left(\mathrm{P}<5 \times 10^{-8}\right)^{27}$.

Despite findings of genome-level association, various studies have rejected the association between blood groups and outcomes of COVID-19. Not all studies report a susceptibility that is high for type $A$ blood and low for type $O$ blood; not all trends are consistent (Table 1). For instance, a multi-institutional study by Latz et al. with $>7,500$ individuals reported a higher risk of infection for type $B$, contrary to the majority indicating a higher susceptibility for those of type $A^{28}$. Consequently, some have rejected this association, considering the conflict between populations as well as the lack of validation ${ }^{29}$. Moreover, some groups highlighted the presence of confounding variables in the relationship between blood groups and host susceptibility to SARS-CoV-2. In particular, the study by Fan et al. discovered a higher susceptibility to COVID-19 in female patients with type A blood, suggesting that a sex difference might be a confounding variable affecting the association ${ }^{30}$. Another study highlighted race as a possible 
confounding variable since Caucasian patients of type A blood had a higher infection rate than type A patients of any other race $^{9}$. Such results suggest that the association, if it exists, may involve more covariates than blood groups and host susceptibility.

As proposed by Boudin et al., variation in the study design may result in the conflict between results. Most cohort studies lack an understanding of patient conditions before COVID-19 related hospital admission, despite its impact on study results. For instance, Boudin et al. highlights the susceptibility of type A individuals towards cardiovascular diseases, which may impact the distribution of $A B O$ blood types in cohort studies studying hospitalized versus non-hospitalized individuals ${ }^{31}$. While the studies may report the $\mathrm{ABO}$ distributions in their respective cohort, they fail to consider the underlying circumstances of the patients. Furthermore, the studies vary in their calculation procedure as well; their results are prone to variation based on the method used to derive their trends. A standardized calculation procedure may help determine whether differences in $p$-values for each blood type are a representation of association with susceptibility to SARS-CoV-2 infections or a result of other factors. Without consistency in such proportionbased studies, there is no validation for the association between ABO types and host susceptibility to SARSCoV-2. While GWAS suggests that the variables are associated at a genomic level, development of a standardized calculation procedure will greatly increase the credibility of proportion-based studies.

\section{Implications of the association}

Despite its ambiguous nature, the association between $A B O$ antigens and host susceptibility to SARS-CoV-2 infections raises numerous implications regarding COVID-19 infections. Initially, the variation in infection rates per country could be partly explained by the proportional differences of blood groups in the population. Based on the prevalence of a particular blood group, a population may be more or less susceptible to SARS-CoV-2 infections. Therefore, public health data may be used as a supplementary resource to generate predictions on the magnitude of a COVID-19 outbreak in a population. Alternatively, identifying genomic associations between the $\mathrm{ABO}$ gene and SARSCoV-2 provides insight into possible mechanisms behind the relationship. Further investigation of blood group antigens and their structures will indicate the significance of their genomic association with pathogens ${ }^{32}$. Additionally, the magnitude to which ABO antigens impact the interaction between ACE2 and SARS-CoV-2 particles may lead to variation in the severity of SARS-CoV-2 disease outcomes ${ }^{24}$. While the report by Latz et al. denied the association between blood type and severity of SARS-CoV-2 outcome, Hoiland et al. indicated that patients of type $A$ and $A B$ were more likely to be critically ill, requiring mechanical ventilation ${ }^{33}$. However, there is a significant lack of interest in the impact of $A B O$ antigens on the severity of SARS-CoV-2 infections, in comparison to that for host-susceptibility. Further research on this topic is recommended for a better understanding of the relationship between ABO antigens and SARS-CoV-2 infections.

\section{CONCLUSION}

Our review highlights the need for a standardized calculation method to determine the magnitude of the association between $\mathrm{ABO}$ blood types and host susceptibility to SARS-CoV-2 infections. While studies examining the relationship have successfully highlighted significant associations, results often vary between similar projects. Deriving p-values per ABO blood group via a unified method will provide insight into whether the variation between cohorts results from study design or represents an association between the variables.

\section{METHODS}

Figure 1 was visualized in UCSF Chimera ${ }^{36}$. Google Scholar was used as the main search engine for the articles, with key phrases including "ABO blood groups", "ABO blood type and respiratory infections", "COVID-19 blood groups", and "blood group susceptibility". Publications were accessed through online journals, then selected based on their implications to COVID-19 and strength of evidence. Their main findings, including $\mathrm{P}$-values on the correlation between the $\mathrm{ABO}$ blood groups and susceptibility to COVID-19 infections, were summarized in Table 1.

\section{ACKNOWLEDGEMENTS}

We thank Dr. Hyojung Ryu for assistance on the visualization of Figure 1 and Sungwon Jeon and Dr. Jong Bhak for advice on the manuscript.

\section{COMPETING INTERESTS}

No competing interests declared.

Received: November 25, 2020

Accepted: June 28, 2021

Published online: July 16, 2021

\section{REFERENCES}

1. Romano, M., Ruggiero, A., Squeglia, F., Maga, Giovanni \& Berisio., R. A Structural View of SARS-CoV-2 RNA Replication Machinery: RNA Synthesis, Proofreading and Final Capping. Cells 9(5): 1267 (2020). 
2. Johns Hopkins University \& Medicine. COVID-19 Dashboard by the Center for Systems Science and Engineering (CSSE) at Johns Hopkins University (JHU). https://coronavirus.jhu.edu/map.html (2021).

3. World Health Organization. MERS situation update, January 2020. http://www.emro.who.int/pandemic-epidemic-diseases/ mers-cov/mers-situation-update-january-2020.html (2020).

4. Centers for Disease Control and Prevention. SARS Basics Fact Sheet. https://www.cdc.gov/sars/about/fs-sars.html (2017).

5. Gilbert, L.G., SARS, MERS and COVID-19-new threats; old lessons. Int. J. Epidemiol 49(3): 726-728 (2020).

6. Liu, Y., Gayle A. A., Wilder-Smith, A. \& Rocklöv J. The reproductive number of COVID-19 is higher compared to SARS coronavirus. J. Travel Med 27(2): taaa021 (2020).

7. Dean, L. Chapter 2 Blood group antigens are surface markers on the red blood cell membrane. Blood Groups and Red Cell Antigens (2). (2005).

8. Liumbruno, M. G., Franchini, M. Beyond immunohaematology: the role of the $A B O$ blood group in human diseases. J. Blood Transfus. 11(4): 491-499 (2013).

9. Leaf, R. K., Al-Samkari, H., Brenner, S. K., Gupta, S. \& Leaf, D. E. ABO phenotype and death in critically ill patients with COVID-19. Br. J. Haematol. 190: e-181-e-232 (2020).

10. De Oliveira, D. M. et al. Blood Group Antigen Recognition via the Group A Streptococcal M Protein Mediates Host Colonization. mBio 8(1): e02237-16, (2017).

11. Cooling, L. Blood Groups in Infection and Host Susceptibility. Clin. Microbiol. Rev 28(3), 801-870 (2015).

12. Dean, L. Chapter 7 The Rh blood group. Blood Groups and Red Cell Antigens (2). (2005).

13. Kaidarova, Z. et al. Blood group A and Rh(D)-negativity are associated with symptomatic West Nile virus infection. Transfusion 56(7): 1699-1706 (2016).

14. Mackenzie, J. S., Fimmel P. J. The effect of ABO blood groups on the incidence of epidemic influenza and on the response to live attenuated and detergent split influenza virus vaccines. J Hyg 80(1): 21-30 (1978).

15. Cheng, Y. et al. ABO Blood Group and Susceptibility to Severe Acute Respiratory Syndrome. JAMA 293(12): 1447-1451 (2005).

16. Guillon P. et al. Inhibition of the interaction between the SARSCoV spike protein and its cellular receptor by anti-histo-blood group antibodies. Glycobiology 18(12): 1085-1093 (2008).

17. Yan, R., Zhang, Y., Li, Y., Xia., Guo, Y. \& Zhou, Q. Structural basis for the recognition of SARS-CoV-2 by full-length human ACE2. Science 367(6485): 1444-1448 (2020).

18. Hoffmann, M. et al. SARS-CoV-2 Cell Entry Depends on ACE2 and TMPRSS2 and Is Blocked by a Clinically Proven Protease Inhibitor. Cell 181: 271-280 (2020).

19. Rossi, G.A., Sacco, O., Mancino, E., Cristiani, L. \& Midulla, F. Differences and similarities between SARS-CoV and SARS-CoV-2: spike receptor-binding domain recognition and host cell infection with support of cellular serine proteases. J. Infect 48: 665-669 (2020).

20. Zhao, J. et al. Relationship between the ABO Blood Group and the COVID-19 Susceptibility. Clin. Infect. Dis doi:10.1093/cid/ ciaa1150 (2020).

21. Zietz, M., Tatonetti, N. P. Associations between blood type and COVID-19 infection, intubation, and death. Nat Commun 11:5761
(2020).

22. $\mathrm{Li}$, J. et al. Association between $A B O$ blood groups and risk of SARS-CoV-2 pneumonia. Br. J. Haematol. 190(1): 24-27 (2020).

23. Barnkob, M. B. et al. Reduced prevalence of SARS-CoV-2 infection in ABO blood group O. Blood Adv. 4(20): 4990-4993 (2020).

24. Wu, Y., Feng, Z., Li, P. \& Yu, Q. Relationship between ABO blood group distribution and clinical characteristics in patients with COVID-19. Clin. Chim. Acta. 509: 220-223 (2020)

25. Göker, H. et al. The effects of blood group types on the risk of COVID-19 infection and its clinical outcome. Turk J Med Sci. 50: 679-683 (2020).

26. Ellinghaus, D. et al. Genomewide Association Study of Severe Covid-19 with Respiratory Failure. NEJM 383: 1522-34 (2020).

27. Roberts, G. H. L. et al. AncestryDNA COVID-19 Host Genetic Study Identifies Three Novel Loci. Preprint at https://www.medrxiv. org/content/10.1101/2020.10.06.20205864v1.full (2020).

28. Latz, C. A. et al. Blood type and outcomes in patients with COVID-19. Ann. Hematol. 1-6 (2020).

29. Khalil, A., Feghali, R. \& Hassoun, M. The Lebanese COVID-19 Cohort; A Challenge for the ABO Blood Group System. Front. Med. 7: 585341 (2020).

30. Fan, Q. et al. Association Between ABO Blood Group System and COVID-19 Susceptibility in Wuhan. Front. Cell. Infect. Microbiol. 10: 404 (2020).

31. Boudin, L., Dutasta, F. Relationship between ABO blood groups and COVID-19: study design matters. Clin. Infect. Dis. ciaa1473 (2020).

32. Ewald, D. R., Summer, S. Blood Type Biochemistry and Human Disease. Wiley Interdiscip. Rev. 8(6): 517-535 (2016).

33. Hoiland, R. L. et al. The association of $A B O$ blood groups with indices of disease severity and multiorgan dysfunction in COVID-19. Blood Adv. 4(20): 4981-4989 (2020).

34. Padhi, S. et al. ABO blood group system is associated with COVID-19 mortality: An epidemiological investigation in the Indian population. Transfus. Clin. Biol. 27(4): 253-258 (2020).

35. Abdollahi, A., Mahmoudi-aliabadi, M., Mehrtash, V., Jafarzadeh, B. \& Salehi, M. The Novel Coronavirus SARS-CoV-2 Vulnerability Association with ABO/Rh Blood Types. IJP 15(3): 156-160 (2020). 36. Pettersen, E. F. et al, UCSF Chimera--a visualization system for exploratory research and analysis. J Comput Chem 2004, 25, (13), 1605-12. 